\title{
A Case of Bladder-Inverted Papilloma after Brachytherapy for Prostate Cancer
}

\author{
Taisuke Ezaki $^{\mathrm{a}} \quad$ Takeo Kosaka $^{\mathrm{a}}$ Shuji Mikami ${ }^{\mathrm{b}} \quad$ Naoto Kaburaki $^{\mathrm{a}}$ \\ Ryuichi Mizuno ${ }^{a}$ Mototsugu Oya ${ }^{a}$ \\ Departments of ${ }^{\mathrm{a}}$ Urology and ${ }^{\mathrm{b}}$ Diagnostic Pathology, Keio University School of Medicine, \\ Tokyo, Japan
}

\section{Key Words}

Inverted papilloma $\cdot$ Brachytherapy $\cdot$ Prostate cancer

\begin{abstract}
An 82-year-old male who presented with the chief complaint of gross hematuria and a history of prostate cancer treated with brachytherapy 6 years previously is described Cystoscopy revealed multiple bladder tumors on the right posterior wall. A transurethral resection of the bladder tumor was performed and a pathological diagnosis of the inverted papilloma was made. To the best of our knowledge, this case is the first report of bladderinverted papilloma after brachytherapy for prostate cancer.

(C) 2014 S. Karger AG, Basel
\end{abstract}

\section{Introduction}

The common causes of hematuria are infection, trauma, prostatic hypertrophy, urolithiasis, urothelial or renal parenchymal malignancy, and glomerular causes. Mucosal irritation, radiation cystitis, and a bladder tumor may cause hematuria in prostate cancer patients after the treatment with brachytherapy [1,2]. Inverted papilloma is a noninvasive urothelial neoplasm and a rare cause of hematuria. We present a case of bladder-inverted papilloma indicated by gross hematuria, which occurred 6 years after brachytherapy for prostate cancer. 


\section{Case Presentation}

An 82-year-old Japanese male with a history of prostate cancer presented to our urology department with the chief complaint of intermittent gross hematuria that had lasted for 1 week. He was not taking any anticoagulant or antiplatelet drugs. He received brachytherapy monotherapy as the definitive treatment for a serum prostate-specific antigen level of 9.21 $\mathrm{ng} / \mathrm{ml}$, a Gleason score of $5(3+2)$, and a clinical $\mathrm{T} 2 \mathrm{~b}$ adenocarcinoma of the prostate treated with 80 iodine-125 implants using a transrectal ultrasound-guided technique. Neither recurrence nor metastasis was detected in the following 6-year period. In the follow-up period, his serum prostate-specific antigen level fell to $0.03 \mathrm{ng} / \mathrm{ml}$.

Urine analysis showed no evidence of pyuria. The patient did not have any lower urinary tract symptoms (LUTS), and the physical examination was unremarkable. Laboratory data were within normal limits, and urine cytology was negative for bladder cancer. Cystoscopy was performed and revealed multiple papillary tumors on the right side of the posterior wall (fig. 1). There was no mucosal edema or erythema that implied radiation-induced cystitis. Computed tomography (CT) showed multiple bladder tumors $<1 \mathrm{~cm}$ in diameter and no abnormal findings (fig. 2). The prostate volume on CT was $25.1 \mathrm{ml}$. The findings suggested the presence of noninvasive bladder cancer.

We performed a transurethral resection of the bladder tumor (TURBT). Multiple nodular tumors found on the right posterior wall were resected and coagulated. After TURBT, the gross hematuria resolved. The TURBT tissue stained by HE showed mildly atypical epithelial proliferation with inverted growth. The basal cell marker p63 definitely stained a twolayered structure. Negativity for MIB-1 with staining in less than $1 \%$ of the tumor cells showed a low proliferative potential. Some tumor cells that stained weakly for p53 were present in a scattered but not diffused pattern. A pathological diagnosis of inverted papilloma was made. The patient is still followed up, and there has been no recurrence of the hematuria or bladder tumors in the 4 months since TURBT.

\section{Discussion}

Inverted papilloma is a rare noninvasive urothelial neoplasm with an endophytic growth pattern covered by an intact layer of histologically and cytologically normal epithelium. The histological feature is the endophytic growth pattern in the lamina propria with cords, trabeculae or nests with variable amounts of intervening stroma, with bland cytologic features and lacking evidence of significant cellular atypia or invasion and is covered by histologically normal urothelium [3].

The immunohistochemical characteristics of inverted papillomas are different from those of urothelial carcinomas. The expression of the proliferation marker Ki-67 (the monoclonal antibody against MIB-1) or the protein product of the tumor suppressor gene p53 (p53 protein) is infrequent in inverted papilloma [4]. We histologically and immunohistochemically diagnosed the tumors as inverted papillomas based on these staining characteristics (fig. 3).

The most commonly reported sites of bladder-inverted papilloma are the bladder neck region (23-41\%) and the trigone (24-35\%), and $0-4.2 \%$ have multiple foci [5]. In the present case, the inverted papilloma was multiple and was found on the right posterior wall of the bladder. It is reported that $5-14 \%$ of the inverted papillomas develop on the posterior wall. The recurrence rate is $1-7 \%$ [5], which is considerably lower than that of low-grade or early-stage urothelial carcinoma. 
To our knowledge, the present case is the first report of a bladder-inverted papilloma after radiation therapy for prostate cancer that was found through evaluation of gross hematuria. Brachytherapy may result in gross hematuria by causing mucosal irritation and friability, neovascularization of the bladder or prostatic urethra, radiation cystitis, and urethral edema or necrosis, but there is no obvious explanation by hematuria workups in the majority of the patients, and it resolves spontaneously in most of them [1]. However, in this case, it is reasonable to conclude that the cause of hematuria was inverted papilloma. Hematuria is the most common symptom of inverted papilloma [5].

The association between radiation therapy and the occurrence of inverted papilloma is unclear. Some have claimed that inflammatory conditions and irritation owing to an enlarged prostate may contribute to the development of inverted papillomas [6, 7]. This could explain the predominance of inverted papillomas in areas potentially exposed to the greatest irritation, the rarity of multifocal lesions, and the very low recurrence rate. After brachytherapy, patients also experience some degree of urinary irritative/obstructive symptomatology. An analysis revealed that grade 3 chronic urinary morbidity was found to occur in $1-3 \%$ of the patients after brachytherapy, which most likely resulted from overirradiation of the bladder base or a persistent inflammatory response in the prostatic urethra [8]. Although our patient had not had previous LUTS, the possibility that asymptomatic irritation or obstruction following brachytherapy induced the inverted papilloma still existed.

Patients who received radiation therapy have a known risk of developing second malignancies. Previous studies showed an increased risk of bladder cancer after radiation therapy including brachytherapy for prostate cancer, and the incidence has been reported to be 1$3 \%$. However, it has been reported that patients with brachytherapy monotherapy have a lower incidence of secondary bladder cancer than those with external beam radiation therapy [9]. Multiplicity and the location of tumors in the present case were not consistent with the general features of inverted papillomas and implied that factors other than LUTS affected the formation of the tumors, but further investigation is necessary to ascertain whether this inverted papilloma was a brachytherapy-induced second neoplasm.

Although inverted papillomas should be regarded as benign neoplasms, their recurrence is not uncommon. Inverted papillomas could also be considered as a risk factor of bladder cancer [5]. Therefore, cystoscopy every 4 months in the first year and every 6 months in the subsequent 3 years or every 6 months in the first 4 years and yearly afterwards are the recommended follow-up periods for patients with inverted papilloma $[5,10]$. In the case presented here, a history of brachytherapy itself might also have been a risk factor of bladder cancer, which was the reason for the careful followed-up of the patient by urinary analysis and cystoscopy.

\section{References}

1 Barker J Jr, Wallner K, Merrick G: Gross hematuria after prostate brachytherapy. Urology 2003;61:408-411.

2 Anderson JF, Swanson DA, Levy LB, et al: Urinary side effects and complications after permanent prostate brachytherapy: the MD Anderson Cancer Center experience. Urology 2009;74:601-605.

-3 Patel P, Reikie BA, Maxwell JP, et al: Long-term clinical outcome of inverted urothelial papilloma including cases with focal papillary pattern: is continuous surveillance necessary? Urology 2013;82:857-860.

4 Jones TD, Zhang S, Lopez-Beltran A, et al: Urothelial carcinoma with an inverted growth pattern can be distinguished from inverted papilloma by fluorescence in situ hybridization, immunohistochemistry, and morphologic analysis. Am J Surg Pathol 2007;31:1861-1867.

5 Picozzi S, Casellato S, Bozzini G, et al: Inverted papilloma of the bladder: a review and an analysis of the recent literature of 365 patients. Urol Oncol 2013;31:1584-1590.

6 Trites AE: Inverted urothelial papilloma: report of two cases. J Urol 1969;101:216-219. 
Case Reports in

Nephrology and

Urology

\begin{tabular}{l|l}
\hline Case Rep Nephrol Urol 2014;4:131-136 \\
\hline DOI: 10.1159/000365142 & $\begin{array}{l}\text { ○ 2014 S. Karger AG, Basel } \\
\text { www.karger.com/cru }\end{array}$ \\
\hline
\end{tabular}

Ezaki et al.: A Case of Bladder-Inverted Papilloma after Brachytherapy for Prostate Cancer

7 Matz LR, Wishart VA, Goodman MA: Inverted urothelial papilloma. Pathology 1974;6:37-44.

-8 Stone NN, Stock RG: Complications following permanent prostate brachytherapy. Eur Urol 2002;41:427433.

-9 Liauw SL, Sylvester JE, Morris CG, et al: Second malignancies after prostate brachytherapy: incidence of bladder and colorectal cancers in patients with 15 years of potential follow-up. Int J Radiat Oncol Biol Phys 2006;66:669-673.

10 Cheng CW, Chan LW, Chan CK, et al: Is surveillance necessary for inverted papilloma in the urinary bladder and urethra? ANZ J Surg 2005;75:213-217.

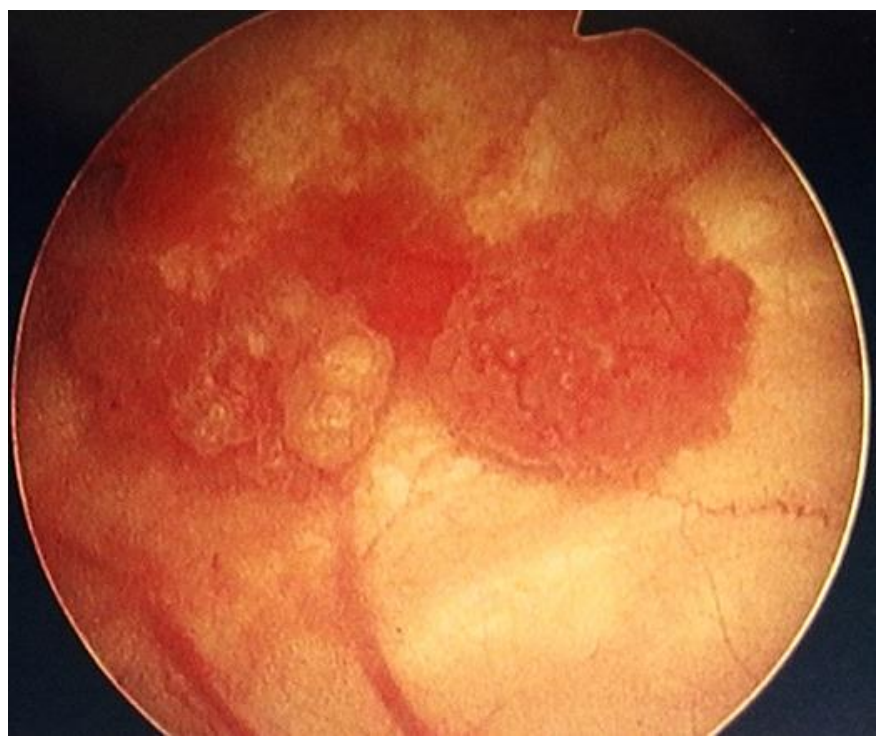

Fig. 1. Image obtained by cystoscopy, showing multiple papillary tumors on the right side of the posterior wall. 
Case Reports in

Nephrology and

Urology
Case Rep Nephrol Urol 2014;4:131-136

DOI: 10.1159/000365142

Ezaki et al.: A Case of Bladder-Inverted Papilloma after Brachytherapy for Prostate

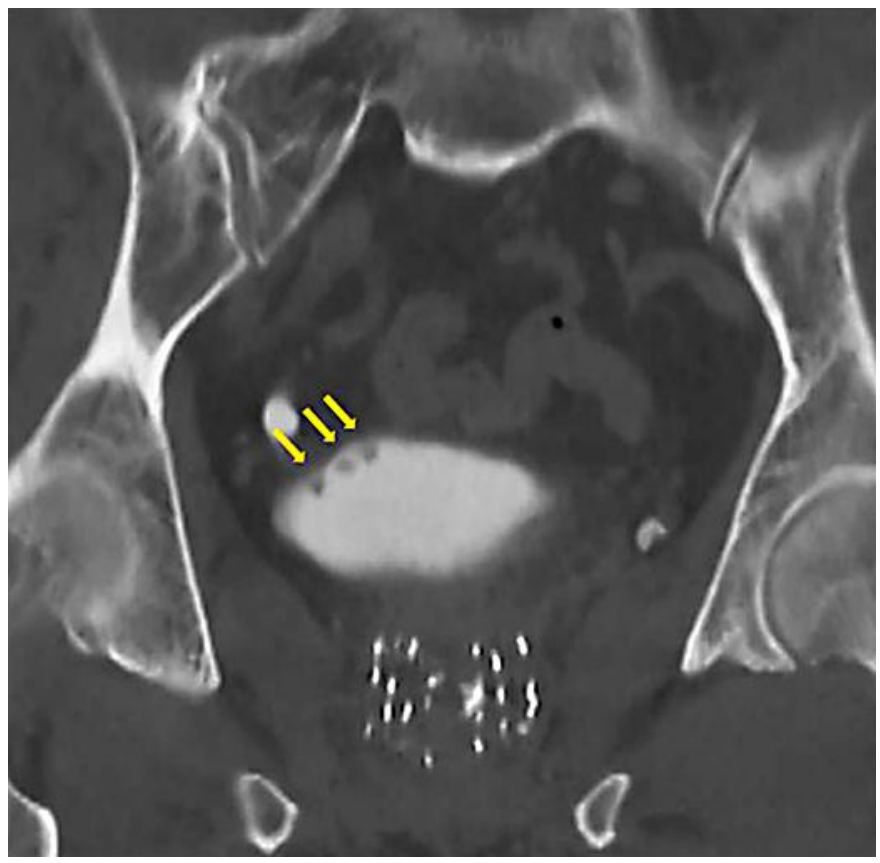

Fig. 2. CT Urography shows multiple tumors $<1 \mathrm{~cm}$ in diameter on the bladder wall. Brachytherapy seeds are implanted in the prostate. 


\begin{tabular}{l|l}
\hline Case Rep Nephrol Urol 2014;4:131-136 \\
\hline DOI: 10.1159/000365142 & $\begin{array}{l}\text { @ 2014 S. Karger AG, Basel } \\
\text { www.karger.com/cru }\end{array}$ \\
\hline
\end{tabular}

Ezaki et al.: A Case of Bladder-Inverted Papilloma after Brachytherapy for Prostate Cancer

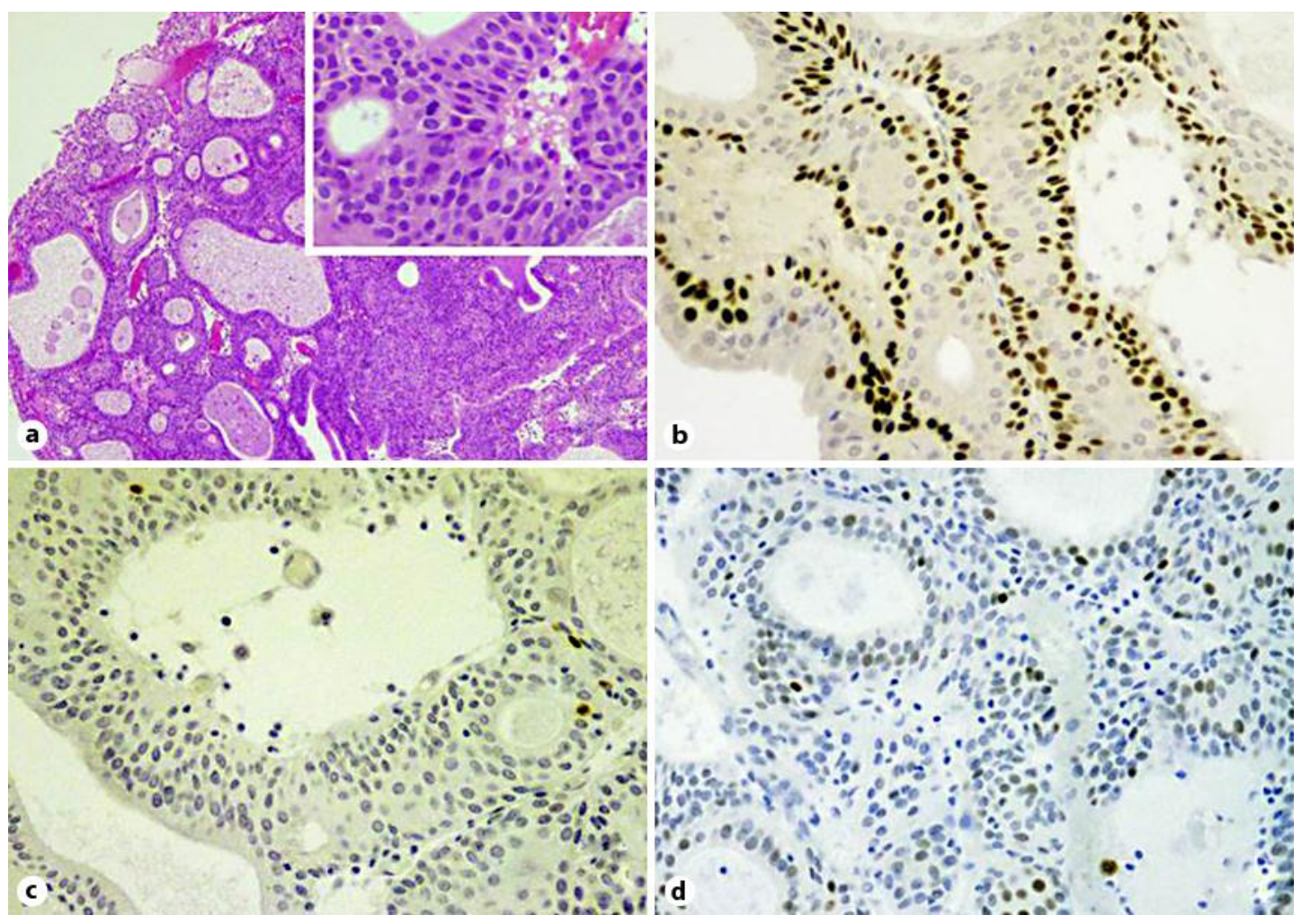

Fig. 3. Paraffin section of TURBT tissue. HE staining (a original magnification, $\times 40$ and $\times 400$ ) and immunohistological staining (b-d original magnification, $\times 200$ ). HE staining (a) showed mildly atypical epithelial proliferation with inverted growth. Definite p63 staining was present on the basal cell layer (b). Negativity or slight positivity for MIB-1 and p53 (c, d). 